I El Colegio de México (Colmex), Centro de Estudios Sociológicos,

Ciudad de México, México

msaavedra@colmex.mx

https://orcid.org/o0oo-0003-4353-0514

Marco Estrada Saavedra'

\title{
EL CONCEPTO SISTÉMICO DE LO POLÍTICO. UN ESBOZO
}

A Irene Álvarez

-¡Miren!, no sean pendejos, la única postura política de peso es no votar jamás. Si todos los votantes se pusieran de acuerdo para no ir a las urnas en un momento dado, entonces sí, ¡qué bárbaro!, sobrevendría sin más el desmantelamiento del mentidero infame al que a base de habladas, dizque muy justicieras, nos quieren empujar, y luego así las cosas ¿a quién pondrían entonces de nueva autoridad?, ¿seguiría el que ya estaba?, ¿o a la fuerza pondrían a tal o cual?, ¿o entre ellos mismos se harían pelotas solos? En fin, de eso se trata: sembrar la confusión, que se peleen entre ellos solamente y que el pueblo, mirando el espectáculo, se dé cuenta por dónde va la cosa.

Daniel Sada

\section{INTRODUCCIÓN}

Desde mis primeros trabajos y hasta la fecha, la relación entre la acción colectiva y la política ha ocupado buena parte de mis investigaciones (Estrada Saavedra, I995, I997, 2007, 20I5, 2016). Si tuviera que sintetizar en una pregunta el problema teórico que encuadra esta relación, la formularía de la siguiente manera: ¿en qué consiste la dimensión política de los movimientos sociales? Me gustaría tomar este interrogante como punto de partida para delinear una sociología de lo político en términos de la teoría de los sistemas sociales de Niklas Luhmann, que contribuya a una comprensión descentrada de la política y la aprehensión de su rica y variada fenomenología.

En vista a este objetivo, revisaré cómo los paradigmas centrales en el estudio de la acción colectiva y los movimientos sociales han tratado su carácter político. Mi tesis es que estos paradigmas empobrecen las dimensiones políticas del fenómeno al partir de una supuesta diferencia ontológica entre el Estado y la sociedad. En seguida, esbozaré algunos puntos centrales de la sociología política de Niklas Luhmann con el fin de ofrecer una concepción compleja y dinámica del sistema político y, con ello, de la variedad de relaciones 
que los movimientos podrían entablar, en principio, con dicho sistema en diferentes niveles. Sin embargo, como la concepción del sistema político del sociólogo alemán aún conserva una fuerte impronta estato-céntrica, propondré introducir la distinción la política/lo político con base en la recuperación de las discusiones en la filosofía política contemporánea al respecto. Posteriormente, traduciré estos debates en términos sistémicos.

El diálogo que propongo entre la antropología del Estado, la teoría de sistemas y la filosofía política es posible en la medida en que todas ellas se caracterizan por su impronta postontológica. En efecto, parten de la diferencia y no de la unidad para construir objetos complejos, hétero jerárquicos, descentrados, autorreferentes, contingentes, relacionales, radicalmente temporales, multicausales y abiertos al cambio. Para este tipo de objetos, los principios de la identidad y contradicción de la lógica no se aplican (Clam, 2002; Gripp-Hagelstange, 2004; y Marchart, 2013). ${ }^{\mathrm{I}}$

\section{LAS DIMENSIONES POLÍTICAS DE LOS MOVIMIENTOS SOCIALES}

El estudio de los movimientos sociales y la acción colectiva ha orbitado alrededor de dos paradigmas: el de la estrategia y el de la identidad (Cohen, I985; Cohen \& Arato, 2000). Se trata de paradigmas (Kuhn, I979) en tres sentidos importantes: en primer lugar, porque diferentes comunidades científicas (escuelas o tradiciones) elaboran su trabajo tomándolos como modelos convencionales de hacer investigación empírica y teórica. En segundo término, a dichos paradigmas les subyacen tipos de acción y racionalidad social distintivos, a saber: la acción social con arreglo a fines y la acción social con arreglo a valores, para utilizar los términos clásicos de Max Weber. Y, en tercera instancia, en torno a estos tipos de acción se han elaborado diversos modelos teóricos de los movimientos y la acción colectiva. ${ }^{2}$

Para los enfoques agrupados en el paradigma de la estrategia - la movilización de recursos, los constreñimientos estructurales, la estructura política de oportunidades o la dinámica de la contienda política (Olson, I965; Tilly, I978; Oberschall, I973; Tarrow, I997; y McAdam, Tarrow \& Tilly, 200I) -, la dimensión política de los movimientos sociales surge en el momento del establecimiento de la interacción contenciosa entre el actor movilizado y las autoridades públicas con el fin de que el primero sea reconocido como un actor legítimo del sistema político. De este modo, el movimiento puede influir en los procesos y mecanismos del sistema que afectan sus intereses. Se trata de la concepción convencional de la política como la de la lucha por el poder y los recursos en el sistema político.

Por su parte, los enfoques aglutinados en el paradigma de la identidad - los genéricamente denominados como los de los nuevos movimientos sociales (Alberoni, I984; Offe, I985, I988; Touraine, I974, I995, I999, 2002; Melucci, I989, I996; Habermas, I988, y I989) - no consideran la dimensión política de los 
movimientos sociales como relevante. Tienen una concepción "metapolítica" de ellos ya que no es el sistema político-institucional en donde se encuentra el "significado" de las luchas de los movimientos sociales. ${ }^{3}$ De hecho, este paradigma no encuentra movimientos sociales como tales en las movilizaciones y protestas políticas, sino sólo "comportamientos colectivos" en busca de representación de intereses políticos. En otras palabras, los considera como un modo inferior de acción colectiva. La "metapolítica" de este paradigma se expresa en que los "auténticos" movimientos sociales orientan sus acciones hacia la defensa de la "identidad" en la sociedad compleja con el fin de la realización de la "autonomía del sujeto", en conflicto con la dominación social. Como en este tipo de sociedad se considera a la información como el recurso existente más importante, la dominación social es entendida en términos culturales.

\section{EL MURO ONTOLÓGICO ENTRE EL ESTADO Y LA SOCIEDAD}

Al parecer nos enfrentamos con dos versiones irreconciliables sobre las dimensiones políticas de los movimientos sociales. Sin embargo, ¿son realmente opuestas? A pesar de sus diferencias evidentes, en mi opinión, ambos paradigmas comparten más de lo que se sospecha, por lo que convergen en sus respectivas formas de reduccionismo en la comprensión de las dimensiones políticas de los movimientos sociales.

Además de sus fundamentos accionalistas, que aquí no voy a tematizar, ${ }^{4}$ ambos paradigmas abrigan una concepción similar de la sociedad moderna, a saber: conformada por subsistemas especializados con racionalidades propias: sociedad, Estado y economía. El quid no es que esta concepción sea errónea - ya que no lo es necesariamente -, sino que los presupuestos ontológicos con los que entienden esas divisiones y sus efectos prácticos en las operaciones teórico-metodológicas en la investigación empírica tienden a reificarlos. Así, entonces, estrechan nuestro entendimiento de las dimensiones políticas de los movimientos sociales.

Con base en las aportaciones de la antropología del Estado (Sharma \& Gupta, 2006; Das \& Pole, 2004; Joseph \& Nungent, I994; Krupa \& Nugent, 2015; Migdal, 20I I; Agudo Sanchíz \& Estrada Saavedra, 20I I, 20I4; y Agudo Sanchíz, Estrada Saavedra \& Braig, 20I7), propongo en seguida un conjunto de críticas que dan cuenta de los problemas de esta distinción que opera como el punto ciego de los estudiosos de los movimientos sociales.

Estos paradigmas suponen que hay una suerte de frontera ontológica entre el Estado (sistema político) y la sociedad (civil), que crea espacios estancos autocontenidos (Mitchell, I99I). En estos predominan tipos de racionalidad propios: la instrumental y la valorativa-normativa. Así, cuando un actor cruza esta frontera, por alguna razón, asume la racionalidad y las cualidades del terreno al que ha ingresado. Por ejemplo, el movimiento social asume, en ese tránsito, su dimensión política gracias a la interacción contenciosa con la autoridad pú- 
blica, en su versión positiva, o a que no logra trascender la lógica del sistema político-institucional, por lo que sólo defendería intereses particulares, de acuerdo con la versión negativa. Lo anterior deja ver una concepción lineal y topográfica de la política, es decir, como una actividad en una dirección y en un espacio específicos. El Estado es representado como una entidad cualitativamente diferente a la "sociedad". Sus instituciones no serían semejantes a las de ésta última. No se encontrarían en su mismo nivel, sino por "encima" debido al potencial de violencia física con el que cuentan para lograr la obediencia sobre los ciudadanos e imponer su autoridad, a través de la acción coordinada de un conjunto de aparatos burocráticos encargados de hacer valer leyes, políticas y ordenamientos en las más variadas esferas de la vida social a lo largo y ancho de un territorio reclamado por el Estado como suyo. De tal suerte, el Estado es visto como un actor unitario, centralizado y autónomo. Lo anterior conduce a observar la protesta social como una manifestación de conflictos entre la sociedad y el Estado. Debido a ello, se homogeniza también al movimiento social como una unidad de acción, fuertemente cohesionada, con identidad, intereses, creencias, voluntad y motivaciones compartidas por todos sus integrantes en oposición al Estado. Sólo así se puede entender que esa relación conflictiva se presente como una lucha entre el sujeto y el sistema, como expresaría Alain Touraine (2002), o el conflicto entre actores del mundo de vida y el sistema, como lo denominaría Jürgen Habermas (I988). Finalmente, el supuesto de la división ontológica entre Estado y sociedad conduce a estandarizar el tipo de conflictos y relaciones que los movimientos entablan con la variedad de actores del sistema político. Actores políticos que son tan distintos como, por ejemplo, los presidentes municipales, las élites tecnocráticas, los burócratas a nivel de calle, los empleados públicos encargados de la aplicación de programas sociales a nivel local, los intermediarios políticos en relaciones clientelares, los partidos políticos, los parlamentarios, los cabilderos, los líderes de opinión pública, los grupos de presión, los medios de comunicación, las organizaciones corporativas oficialistas, los organismos autónomos del Estado (como las comisiones de defensa de derechos humanos o los órganos electorales), la policía, el ejército, etcétera. Esta variedad de actores en el entorno interno del sistema político es simplificada, de manera burda, con la etiqueta "autoridades públicas" o "sistema político-institucional”, así como la hipótesis problemática del enderezamiento de sus acciones de acuerdo con una única racionalidad compartida.

En síntesis, si se cuestiona la realidad de la demarcación ontológica de la división Estado/sociedad y la hipótesis de la existencia de racionalidades correspondientes a cada uno de los términos de esta distinción, entonces podríamos observar las complejas, contingentes y cambiantes relaciones que entablan Estado y movimiento y dar cuenta de cómo los movimientos pueden estar en el Estado y el Estado en la sociedad (es decir, los movimientos). Cuando desencializamos y deconstruimos nuestras nociones de Estado y movimiento, 
ganamos una concepción más compleja y dinámica de ambos y sus relaciones mutuas. Al aplanar (Latour, 20Io) la idea de Estado, se le puede tratar como una fuerza social más (Migdal, 20I I) entre las existentes en la sociedad (e incluso allende las fronteras nacionales), que se encuentran en luchas por ordenar la vida, relaciones, identidades, lealtades, distribución de poder, autoridad y todo tipo de recursos de la población en su conjunto o de un segmento de ella. En una palabra, luchan por hacer valer órdenes sociales de dominación (más o menos extensos, según sea el caso).

Sin embargo, lo anterior no agota las dimensiones políticas de los movimientos sociales. En realidad, con esta propuesta aún se orbitaría alrededor de la concepción politicocéntrica propia de ambos paradigmas (y del sentido común de la mayoría de los científicos sociales). Se necesita dar cuenta, además, de las dimensiones políticas del movimiento antes y después de la confrontación con las autoridades públicas, sistema político o Estado, es decir, en el mundo de vida. ${ }^{5}$ Lo anterior implica reelaborar la misma noción de movimiento social y dar cuenta de su complejidad y heterogeneidad internas para romper con la ilusión de unidad e identidad que propagan ambos paradigmas, por un lado, y reconocer las diferencias, desigualdades y relaciones de poder y conflictos que los estructuran internamente, así como las experiencias de proyectos alternativos de vida individual y colectiva en el mundo de vida, por el otro (Estrada Saavedra, 20I5). Justo aquí encontramos dimensiones de la política que no se pueden simplificar en el mero reconocimiento como actor integrante de la polity ni pensarlas en términos de un conflicto por apoderarse de la historicidad o la reprogramación de códigos societales.

Aún más: hay que tomar en serio el hecho de que muchos conflictos en los que se involucran los movimientos sociales los enfrentan contra oponentes y en arenas distintas a los del sistema político. Por lo tanto, es necesario incluir en el análisis sociológico lógicas, códigos, medios de comunicación simbólicamente generalizados y programas propios de los sistemas funcionales (por ejemplo, el derecho, el arte, la economía, los medios de masas, la religión, etc.) en los que tiene lugar el antagonismo. Inclusive es de anticiparse que el movimiento participa contemporáneamente en conflictos múltiples con actores y organizaciones de diversos sistemas funcionales. Por esta razón, adopta diferentes formas de acción y comunicación (paralela y/o consecutivamente) en la consecución de objetivos heterogéneos y en constante revisión y reajuste.

La crítica de la denominada antropología del Estado a la concepción de una división ontológica entre Estado y sociedad tiene el inconveniente de sugerir que no existe diferencia real entre ambos, sino sólo una dispersión de discursos y prácticas de poder desarticuladas. Concebido así, el Estado correría el riesgo de convertirse en una mera representación mental o ideológica (Abrams, I988), y la dominación política en asimetrías de poder discretas y volátiles. Una conclusión semejante sería inaceptable. El momento de descentramiento y de- 
construcción del Estado promovido por esta antropología debe ser llevado a uno de reconstrucción, mas sin caer en las ilusiones del Estado como un aparato unitario, autónomo e hiperracionalizado (Agudo Sanchíz, 20I4; Estrada Saavedra, 20I9).

En la sociología política de Niklas Luhmann se pueden encontrar vías prometedoras para llevar a cabo este propósito.

\section{LA POLÍTICA DE LA SOCIEDAD}

En esta sección, esbozo, de manera muy sintética, la descripción de Niklas Luhmann del sistema político. ${ }^{6}$

Como es conocido, el sociólogo alemán concebía a la sociedad moderna como una sociedad funcionalmente diferenciada y compuesta por una pluralidad de sistemas autónomos y especializados en la resolución de problemas societales (Luhmann, I998).?

De acuerdo con el sociólogo de Bielefeld, la función de la política consiste en la "toma de decisiones vinculantes colectivamente" (Luhmann, 2000a: 84). Las decisiones políticas no sujetan a la "sociedad" en su conjunto, sino que se dirigen a y/o crean determinadas "colectividades", a las cuales vinculan y, en caso de resistencia, las obligan a obedecer - en una situación extrema inclusive por medio de la violencia (Nassehi, 2002). En este sentido, el poder es el medio de comunicación simbólicamente generalizado del sistema político. En otras palabras, es el instrumento para elaborar, hacer efectivas y comunicar decisiones políticas (Luhmann, 2000a). La existencia de una enorme variedad de posibles cursos de acción en la política hace necesario el poder. En efecto, lo propio de las decisiones es que son contingentes. Antes de ser tomadas, suponen una pléyade de alternativas más o menos plausibles, más o menos preferibles. Una vez que se han tomado, las decisiones son presentadas como necesarias e ineludibles - astucia que sólo invisibiliza su radical contingencia como una mera selección posible entre muchas otras que han sido descartadas (Luhmann, 2000c, en particular el capítulo 4).

Con el objetivo de lograr su aceptación - sobre todo teniendo en cuenta que en la colectividad política las preferencias político-ideológicas son plurales y contrarias -, el poder contribuye a reducir las alternativas de respuesta a la decisión comunicada - obedecer o desobedecer - y señalar los cursos de acción que los tomadores de decisión en el sistema político prefieren que se sigan. De esta manera, el poder resuelve de continuo el problema de la doble contingencia social en el sistema. Dicho de otro modo, el poder funciona cuando Ego está dispuesto a obedecer voluntariamente a Alter, de tal suerte que establece un vínculo y demuestra su disposición a cooperar en los términos establecidos. El poder abre un abanico de alternativas, por lo que la decisión de obedecer y cooperar es, en consecuencia, contingente. Pero, en última instancia, si la aceptación de obedecer voluntariamente no se consigue, se puede obligar a com- 
portarse de la manera deseada mediante la amenaza del uso de la violencia física. El empleo de la violencia clausura toda posibilidad de decidir de Ego y, por tanto, de colaborar libremente (Luhmann, 20I2). Este es el significado último del carácter colectivamente vinculante de la decisión política. Un "no" a la comunicación de poder puede conducir a que Ego sufra una sanción, que puede ir desde una multa administrativa ${ }^{8}$ hasta el encarcelamiento o la pena de muerte. ${ }^{9}$ Por lo general, el detentador del poder político institucional tiene un gran interés en que sus mandatos sean aceptados de manera libre y voluntaria. De este modo, no sólo fluyen los procesos comunicativos sin mayores escollos, sino que además se refrenda la legitimidad de la autoridad política. La violencia es la ultima ratio de la autoridad para conseguir obediencia. Su aplicación representa, sin embargo, un riesgo para el poder. El refinamiento del simbolismo del poder reside justamente en la amenaza de sanciones negativas, no en el ejercicio de la sanción. La utilización de la coacción física puede ser un signo palpable de la erosión de la legitimidad del poder político.

Por su parte, el código del sistema político tiene la forma gobierno/oposición. ${ }^{\text {Io }}$ Gracias a él, se logra la clausura operativa del sistema político mediante la tecnificación de la comunicación inherente a la política. El gobierno observa la función de la toma de decisiones y la comunicación de órdenes a la administración para su aplicación. A su vez, la oposición supervisa y critica las tareas del gobierno, con lo cual demuestra, al menos discursiva y polémicamente, que existen alternativas a las propuestas y decisiones gubernamentales. La oposición se ocupa, también, de disminuir la asimetría de poder existente entre centro y periferia. ${ }^{\text {II }}$ Así, sirve de zona de contención entre el gobierno y la opinión pública/pueblo/ciudadanía, con lo cual permite incluir temas y actores de la periferia del sistema. "El mismo carácter de 'o esto o aquello' de la codificación [gobierno/oposición] funge como restricción" (Luhmann, 2000a: I02). En efecto, el código restringe las posibilidades de organizar y utilizar el poder, de ubicarse al mismo tiempo en el gobierno y en la oposición o de quiénes y qué temas pueden ser incluidos o excluidos en la toma de decisiones políticas.

Por otro lado, el sistema político está diferenciado, internamente, en tres subsistemas: la política, la administración pública y el público (Luhmann, 200oa: 253ss, 258s y 26oss). La unidad de la diferenciación interna del sistema político es asegurada y representada simbólicamente por el Estado. En el primer subsistema, encontramos las estructuras, procesos y actores de los poderes ejecutivo y legislativo, pero también de los partidos políticos, que en su conjunto cooperan en la elaboración de decisiones políticas. En este sentido, la "política" es la totalidad de "comunicaciones que [sirven] para preparar decisiones colectivamente vinculantes mediante la prueba y evaluación de sus oportunidades de éxito. Esta actividad supone que ella misma no tiene aún un efecto vinculatorio colectivo, pero que, al mismo tiempo, se expone a ser observada y, por ello, a ser parcialmente fijada" (Luhmann, 2000a: 254). 
En la administración pública encontramos, por su parte, el complejo entramado burocrático que se ocupa de hacer valer leyes, normas, reglamentos, ofrecer servicios a la ciudadanía y usuarios, así como aplicar todo tipo de políticas y programas públicos resultado de las decisiones políticas (Luhmann, 20I0, en particular la tercera parte).

Finalmente, mediante el acoplamiento estructural del sistema político con los medios de masas con fines de observación de su entorno (externo e interno), ${ }^{12}$ el público está conformado por las discusiones que informan temáticamente la opinión pública, y los diferentes roles políticos que los ciudadanos pueden asumir en dicho sistema (votante, miembro de asociaciones civiles, manifestante, contribuyente fiscal, beneficiario de programas sociales, peticionario, etcétera). ${ }^{\mathrm{I}}$ En forma de opinión pública, el público funge como filtro de los temas que más preocupan e interesan a la ciudadanía. Temas que la política puede recoger en sus deliberaciones partidistas y parlamentarias y, eventualmente, representar políticamente.

La anterior no es la única forma de diferenciación social del sistema político. Luhmann propone, también, la existencia de la diferenciación centro/ periferia (Luhmann, 2000a: 244Ss). De acuerdo con ésta, en el centro del sistema se encontrarían los poderes ejecutivos y legislativos, ${ }^{\mathrm{I4}}$ la burocracia estatal y los partidos políticos, mientras que en su periferia hallaríamos la ciudadanía, la opinión pública, los movimientos de protesta, ${ }^{15}$ las organizaciones no gubernamentales, los actores de la sociedad civil, los grupos de presión e interés, entre otros.

Para los fines de nuestra discusión, el valor heurístico de las diferenciaciones política/administración/público y centro/periferia consiste en que nos permite observar qué estructuras, procesos y actores políticos participan, de manera más directa, en la función del sistema político. Gracias a ello podemos registrar las variaciones y dinámicas de las relaciones de poder al interior del sistema (Luhmann, 2000a: 255ss) y tomar en cuenta las diferentes cadenas de comunicación que intervienen, directa o indirectamente, en la elaboración de decisiones políticas.

Como todo sistema social, el político traza, de manera autónoma, su frontera con su entorno al producir y seleccionar el tipo de comunicaciones que le es propio- las decisiones políticas - y contribuye a su autopoiesis. En este sentido, Luhmann considera como político todo fenómeno involucrado, directa o indirectamente, en la elaboración de decisiones colectivamente vinculantes. Por lo tanto, el proceso de su producción puede iniciar, en principio, en cualquier punto del centro o la periferia del sistema político o en cualquiera de sus tres subsistemas especializados. Existe, entonces, un doble "circuito de circulación del poder": uno formal (desde el centro e institucional) y, otro, informal (desde la periferia y no institucional) (Luhmann, 200oa: 258ss y 264). ${ }^{16}$ De este modo, el poder asume una forma diferente si inicia su circulación en el centro o la peri- 
feria, en la administración pública o la opinión pública del sistema. Por ejemplo, un movimiento social (periferia) puede elevar, mediante protestas, demandas (a favor de la protección de la naturaleza o los derechos humanos). Eventualmente, éstas son registradas y discutidas temáticamente por la opinión pública creando un clima de presión sobre partidos de oposición, políticos, parlamentarios y gobierno, que conduzca a tomar una decisión política al respecto. Decisión que asumiría la forma de una política o programa público, que la burocracia deberá implementar. Puede suceder, en cambio y para variar el ejemplo desde otro sentido de este circuito, que la administración pública - el ministerio de comunicaciones y transportes - detecte que, debido al aumento del volumen del tráfico aéreo, la capacidad aeroportuaria del país resulta insuficiente para responder a las necesidades de transporte de personas y mercancías, por lo que propone al ministro de dicha cartera un plan de modernización de aeropuertos. El gobierno buscaría implementar dicho plan. Esto requiere, sin embargo, convencer al público (ciudadanía) de la conveniencia del mismo. A la par, el gobierno negocia su realización y autorización con la oposición en el parlamento. Dado el caso, se toma una decisión política en este sentido.

Esta concepción, rompe dos tipos de ilusiones: la estatista o elitista, que presupone que el poder político se originaría en la punta de la pirámide (centro) y descendería a su base (periferia); y, por el otro lado, la ilusión republicana, que alega que el poder político provendría "del pueblo", que, por medio de mecanismos de delegación y representación, facultaría a políticos y funcionarios a implementar las decisiones populares. Con esta perspectiva descentrada, se refleja de manera más adecuada la complejidad de la política.

Una consecuencia [de lo anterior] es que la localización del poder cambia de situación a situación y que únicamente puede ser observado y calculado en el mismo sistema. Esto es así, porque se debe calcular constantemente la distinción poder formal/informal, por lo que la comunicación (escrita y oral) debe ser leída, correspondientemente, de manera doble. Sobre todo, ha de establecerse una observación de segundo orden, de tal modo que cada observador puede tomar en cuenta que está siendo observado y cómo está siendo observado. Y, no por último y complicando aún más la situación, en este sistema de vinculaciones se utiliza también la distinción inclusión/exclusión (Luhmann, 2000a: 260).

Si partimos de lo anterior, es posible pensar la decisión y el poder políticos como flujos comunicativos - por tanto, creadores de formas de lo social -, que circulan en su proceso de elaboración de manera horizontal, vertical, central, periférica, espiral, contradictoria y/o intermitente. Todo ello sucede en lugares y momentos múltiples que no están, necesariamente, coordinados y sincronizados entre sí ni dirigidos o supervisados centralmente. ${ }^{\text {I7 }}$ Por tanto, los efectos de estos flujos de decisión y poder - es decir, de condicionar las selecciones y acciones de Ego - tienen alcances y consecuencias diferenciados e inesperados. 


\section{LA FILOSOFÍA DE LO POLÍTICO}

Si bien la sociología política de Niklas Luhmann ofrece una ambiciosa y compleja descripción del sistema político de la sociedad, no deja de tener un importante sesgo estato-céntrico al asumir, de manera poco crítica, la semántica establecida en la evolución de la (auto)descripción del sistema mismo. Esto conlleva el inconveniente de la sobredeterminación del denominado "centro" del sistema como el espacio de las operaciones en el que la función de la política se realiza de manera fundamental. En efecto, a pesar de que al Estado no se le otorga un estatus especial en esta sociología, no es fácil quitarse la impresión de que él sigue representando la unidad y totalidad del sistema debido a que dispone del monopolio de la violencia legítima. Por esta razón, la "periferia" y los subsistemas "no centrales" del sistema político no son considerados como igualmente relevantes en su contribución al cumplimiento de la función societal de la política. No obstante, si se toma en serio la exigencia de la universalidad de la teoría de sistemas, entonces, "no hay por qué dar prioridad al centro político sobre la periferia política en la hétero descripción científica del sistema político" (Hellmann, 2005: 45).

Aún más, tampoco habría que hipostasiar la lógica de la diferenciación funcional como la única perspectiva relevante para aprehender la constitución de los fenómenos políticos en y fuera del sistema político. Hay que tomar en cuenta, también y dependiendo del caso a estudiar y la pregunta de investigación, las lógicas de la diferenciación segmentaria, estratificada, centro/periferia o inclusión/exclusión (Holz, 2003; Lehmann, 2003). ${ }^{18}$

Lo anterior nos obliga a ser más exigentes que Luhmann cuando afirma, con toda razón, que:

Cuando la autopoiesis del poder ha logrado establecerse y, en consecuencia, se puede contar con la existencia de diferencias de poder, entonces otras comunicaciones pueden poseer también capacidad de enlace, aunque no participen directamente en la autopoiesis del sistema [...] [En consecuencia], no todas las operaciones políticas versan sobre el manejo y la reproducción del poder político. Muchas, quizás la mayoría de ellas, tienen que ver con ello sólo de manera indirecta (Luhmann, 2000a: 90).

Si esto es así, ¿cuál es el alcance real de los rendimientos de la cooperación del código, el medio de comunicación y los programas en la producción de la función de la política, en el entorno interno de este sistema especializado? ¿En qué sentido se pueden considerar como políticas a las comunicaciones que sólo tangencialmente contribuyen a la elaboración de decisiones políticas? ¿Se podría afirmar con propiedad que, en el sistema político, hay comunicaciones "más" políticas que otras? ¿A qué se referiría este "más" y cómo dar cuenta, heurísticamente, de estos supuestos grados de "politicidad"? Estaríamos hablando de comunicaciones que, sin contribuir formalmente en la elaboración de las decisiones políticas - por lo que no se les puede considerar vinculantes 
colectivamente -, sin embargo, no se les puede dejar de considerar como significativas políticamente por sus efectos en y fuera del sistema político.

Para atacar este problema, propongo trazar, a manera de reentry, una distinción al interior del concepto sistémico de la política y crear la forma la política/ lo político. Con el fin de perfilar el contenido del lado derecho de esta distinción, echaré mano, a continuación, de las discusiones sobre lo político en la filosofía política contemporánea (Arendt, I985, I992, I993, I994; Badiou, I988, I985; Castoriadis, 2008, 200I, I995, I989; Laclau, 20I5, 20I4, 2008; Laclau \& Mouffe, 20I I; Lefort, I986, I 99 I, I992, I999; Rancière, 2002, 2004, 2007; Vollrath, I977, I987, 2003).' ${ }^{\text {9 }}$

Pero antes de esto, conviene escuchar lo que Luhmann tiene que decir sobre la propuesta de introducir una distinción en el concepto sistémico de la política. Sin rodeos, considera "la distinción entre 'la política' y lo 'político' como artificial. Es un síntoma de los intentos de rescatar pesos muertos de la filosofía política de Europa en una época, en la que esta filosofía se enfrenta con estructuras sociales transformadas radicalmente" (citado en Vollrath, 2003: 2I). ${ }^{20}$ Es verdad que en varios de estos filósofos, no en todos, hay una relectura y recuperación de la filosofía clásica. Pero su objetivo no consiste en reanimar la experiencia de la polis ateniense en las condiciones actuales - empresa, por lo demás, fútil - ${ }^{2 \mathrm{I}}$ sino en encontrar herramientas (figuras de pensamiento) para dar cuenta de los déficits con que se aborda en la filosofía ${ }^{22}$ y la ciencia el fenómeno político, la creciente desafección política entre la ciudadanía y, sobre todo, tratar de responder filosófica y prácticamente a la transformación presente de las democracias liberales en regímenes "postdemocráticos" y "postpolíticos". ${ }^{23}$ En una palabra, estos filósofos buscan encarar el supuesto exilio de la política en nuestros sistemas políticos contemporáneos.

Quizás ésta intención político-filosófica no alcanzaría a justificar la introducción de la distinción política/político en la sociología política de Niklas Luhmann. La razón central de la utilización del pensamiento de la "diferencia política" consiste, fundamentalmente, en que en la teoría sistémica hay suficientes problemas, ambigüedades y puntos ciegos sobre la determinación de la política que, para su estudio empírico, en general, y de los movimientos sociales, en particular, se ha vuelto ineludible reevaluarla y recalibrarla -"sin consideración a su arquitectura”- para volverla un instrumento de observación más fino y potente. Aún más, el tufillo "evolucionista", en el sentido más peyorativo del término, de la teoría de sistemas conduce a Luhmann a no hacer ninguna consideración sustancial sobre regímenes políticos diferentes al de la democracia liberal representativa, el cual sirve de modelo para esbozar el sistema político de la sociedad global. Este estrechamiento de la diversidad de regímenes políticos a nivel mundial se paga al precio de ignorar cómo funciona realmente la política en las diferentes regiones del orbe.

Dicho lo anterior, regresemos al argumento central de esta sección. En las obras de estos filósofos - al menos en relación con lo político -, no se pre- 
tende desarrollar un pensamiento sistemático o una teoría acabada. Inclusive su filosofía puede describirse como "anti sistemática" o "asistemática", porque se considera que, salvo en regímenes totalitarios, lo político no puede clausurarse, lo cual se reflejaría en el talante no sistemático de sus respectivas filosofías (Bröckling \& Feustel, 20ıо; y Bedorf, 2010).

Más allá de sus diferencias sobre la determinación concreta de lo político - ya sea como un modo especial de discurso o práctica, ${ }^{24}$ un fenómeno con un espacio social propio, ${ }^{25}$ un carácter de evento extraordinario e irruptor, ${ }^{26}$ un criterio normativo, ${ }^{27}$ un acto fundacional ${ }^{28}$ o un tipo singular de relación ${ }^{29}-$, lo que tienen en común estás filosofías de la "diferencia política” (Bedorf \& Röttgers, 20I0 $)^{30}$ es que caracterizan lo político por su capacidad generativa de producir su propio espacio fenoménico, tanto al interior como al exterior de las instituciones políticas. Asimismo, enfatizan su naturaleza antagónica en el sentido de iniciar conflictos que redefinen la figura y sentido de relaciones sociales, la distribución de recursos socialmente valiosos, las identidades individuales y colectivas e, inclusive, las formas de hacer, pensar o sentir de actores y colectividades. Estos filósofos identifican la política con el funcionamiento normal y cotidiano de la administración pública, el debate parlamentario, las luchas partidistas por el ejercicio del poder, las discusiones públicas o la participación electoral. En comparación con lo anterior, lo político interrumpe la normalidad de lo políticamente instituido. En otras palabras, es el momento instituyente que crea formas nuevas de lo social y, por tanto, de ordenar la política. Para estos pensadores, lo político revela, a manera de dispositivo disruptivo, la contingencia del orden sociopolítico, es decir, su carencia de fundamentos naturales, necesarios o racionales, que es lo mismo que decir: su base en la dominación. En este sentido, como la política carece de esencia, en principio cualquier tema, relación o identidad social puede ser politizable (o despotizable). Esto significa, además, que no está definido de antemano quién puede ser o no un sujeto político. De tal suerte, las fronteras de lo social y lo político se vuelven ambiguas y pueden ser dislocadas y reorganizadas

Para una recuperación productiva de las discusiones anteriores, hay que tomar conciencia de que, por razones propias de las prácticas de argumentación en la filosofía, la distinción la política/lo político es tratada como una dicotomía. Además, en tanto que la filosofía es considerada como una praxis política, en el sentido de ser un pensamiento sobre el sentido del estar y ser en el mundo y las formas de convivencia con los otros, se utiliza la distinción como un criterio práctico-normativo. En efecto, la retórica de la oposición conceptual conduce a hipostasiar con frecuencia lo político como la experiencia auténtica de la praxis política y, en consecuencia, a observar en la política su perversión, decadencia o corrupción. Por último, ante el espectáculo de la normalidad política realmente existente en nuestras sociedades, los filósofos destacan, de manera polémica, el carácter extra-ordinario, mesiánico y hasta (proto) revolucionario de lo político. 
Para atemperar algunos de los anteriores excesos, se requiere someter las intuiciones de la filosofía de lo político a una crítica de su "razón escolástica" (Bourdieu, I 999) y colocarlas en la complejidad del mundo social como el sustrato sociohistórico que media, condiciona y organiza las disputas y los combates políticos, así como las prácticas y las formas de pensar y evaluar de los actores sociales involucrados en ellos (Eribon, 2017: I I7-I46; Bourdieu, I 997: I I5-I 25).

\section{EL CONCEPTO SISTÉMICO DE LO POLÍTICO}

En esta última sección quiero traducir, al lenguaje sistémico, las figuras de pensamiento de la filosofía de lo político para hacerlas productivas para la sociología política, en general y el estudio de los movimientos sociales, en particular.

Si partimos de la distinción la política/lo político, del lado izquierdo de la forma se ubican el conjunto de comunicaciones producidas de acuerdo con la lógica operativa del código, el medio de comunicación y los programas del sistema político. En el flanco derecho, se encuentran las comunicaciones que, si bien están también orientadas a "influir en la toma de decisiones políticas", no se ajustan, en sentido estricto, a la lógica sistémico-funcional de la política o, inclusive, se configuran de acuerdo con otras lógicas de diferenciación social. En este punto conviene seguir el concepto de participación política de Huntington y Nelson - "la actividad de ciudadanos particulares orientada a influir en la toma de decisiones gubernamentales" (I976: 4) - a razón de que incluye la participación autónoma individual y colectiva, la corporativamente movilizada, la legal e ilegal, la pacífica y la violenta, la que confirma la legitimidad del régimen y la que busca subvertirlo revolucionariamente. Hay que enfatizar, no obstante, que la "influencia" en la toma de decisiones incluye más que la "esfera gubernamental" que mencionan estos autores. Se ha de considerar tanto la influencia en alguno de los subsistemas de la triada política-administración-público como también, y si es el caso, en otros sistemas funcionales y sus respectivas organizaciones. Todos ellos son espacios en los que puede ejercerse esta "influencia" y en los que se encuentran eventuales oponentes y aliados. En efecto, piénsese en un grupo de pobladores que protesta en contra de la explotación de minerales de una empresa en las cercanías de su entorno natural; o en un colectivo que quiere censurar una cinta cinematográfica o una exposición de arte por considerar que ofende su moral o convicciones religiosas. $\mathrm{O}$, para poner otro ejemplo, imagínese a activistas que se enfrentan en contra de laboratorios científicos en los que se experimenta con primates. En todos estos casos, hay que considerar siempre la lógica de los sistemas económico, del arte o la ciencia, respectivamente, junto con la del sistema político.

Como configuran la unidad de la diferencia de una forma, la política y lo político comparten el poder como su medio de comunicación. Pero, las comu- 
nicaciones de lo político se hallan, desde el punto de vista sistémico-funcional, más indeterminadas y polémicamente más abiertas que las de la política. Las bifurcaciones binarias del código (gobierno/oposición), el medio (poder/no poder) ${ }^{31}$ y los programas (derecha/izquierda) del sistema político son desestabilizadas por lo político al no reducir sus opciones a la lógica tecnificada del "o esto o lo otro". Lo político se caracteriza por transgredir y desplazar los márgenes de la inclusión/exclusión del orden político-sistémico y, en ciertos casos, pretender inclusive subvertirlo al proponer una nueva gramática para trazar distinciones, que sean la base de futuras decisiones colectivamente vinculantes.

Si bien el poder está presente, prácticamente, en todo sistema social (interacción, organización o sistema de protesta), únicamente en el sistema político opera como su medio de comunicación simbólicamente generalizado. Es allí donde sistemáticamente amplía y estructura el rango de las comunicaciones (las decisiones políticas) más allá de los actores presentes y en situaciones múltiples, variadas, aún desconocidas y por venir. En este sistema, el poder condiciona las motivaciones y selecciones de los actores políticos y eleva las probabilidades de aceptación de la comunicación, a pesar de la incomodidad que pueda producir su contenido en sus destinatarios (Luhmann, I998: I9oss).

El medio de comunicación es un lenguaje híper especializado que permite que las comunicaciones que se elaboran con base en él sean significativas para el sistema funcional en cuestión, es decir, que no sean consideradas como "ruido" y tengan, en cambio, capacidad de enlace con los flujos comunicativos sistémicos. Para el caso que nos ocupa, esta capacidad supone que la comunicación de lo político pueda, eventualmente, participar o influir en la configuración de las tomas de decisiones políticas - aun cuando la constitución de sus comunicaciones y acciones no estén (del todo) tecnificadas, por su grado de indeterminación, y los canales que se utilizan para este objetivo violenten la lógica y las expectativas institucionales.

En este sentido, una de las fuentes de poder - pero también de debilidad - de lo político es la alta contingencia que introduce en los procesos comunicativos institucionales. Esta contingencia es percibida como disruptiva en la medida en que suspende la relativa normalidad de las cadenas de comunicación de decisiones políticas. Para los procesos e integrantes de la polity, las demandas contestarias provenientes del espacio de lo político resultan "maximalistas", "poco realistas", "no negociables", "irresponsables”, en otras palabras, "intratables". Esta contingencia irruptora asume la forma de un conflicto, es decir, un sistema social que se reproduce con base en una doble contingencia negativa (Luhmann, I987: 530-538). El conflicto es la comunicación de contradicciones, de tal suerte que se endereza a defraudar continuamente las expectativas de la contraparte. La relación entre Ego y Alter se configura en torno a la rivalidad y la voluntad de hacerse daño entre sí. Por esta razón, la forma principal de comunicación de lo político es la negación. 
En relación con el poder como medio de comunicación, lo político puede entenderse como un "parásito" sistémico. Me explico. El mundo es un horizonte infinito de posibilidades. El sentido es el medio de la reducción y el manejo de su complejidad e inabarcabilidad gracias al uso de, por ejemplo, tipificaciones, esquematismos, codificaciones o programaciones. Las especificaciones de sentido se basan, esencialmente, en el trazo de distinciones, o sea, en la creación de diferencias. Si bien es cierto que estas distinciones son artificiales y no reflejan el sustrato de la realidad mundana son, sin embargo, el principio que permite construir enlaces ulteriores plenos de sentido o cadenas comunicativas mediante la exclusión de otras posibilidades de constitución de sentido, vivencia, experiencia, comunicación y acción. La figura del "parásito" recuerda, precisamente, la arbitrariedad del origen de esas distinciones instituidas. Su presencia irruptora disloca la lógica binaria (la forma con dos lados) con la que opera el sistema (en nuestro caso, el sistema político) trayendo al juego al tercer excluido, es decir, a las posibilidades anteriormente descartadas u otras no consideradas previamente (cfr. Luhmann, I987: I22ss y 285).

El conflicto, que la irrupción de lo político inaugura, desnaturaliza la normalidad imperante y devela la arbitrariedad sobre la que se basa el orden de la política: la dominación. De este modo, lo político abre un espacio de ponderación de otras posibilidades de concebir y organizar lo social y de modificar expectativas institucionalizadas e iniciar procesos de aprendizaje.

Los conflictos disuelven la seguridad de la expectativa, desmontan la complejidad reducida y muestran que es posible más que lo meramente actualizado hasta ahora [...] [El] conflicto retrae al sistema, a la vez, a su estado originario: la complejidad reducida - como complejidad propia - se desgaja (Bonacker, 2005: 273)..$^{32}$

Por otro lado, lo político supone un proyecto alternativo de un modelo de organización colectiva de la sociedad (o, al menos, de un segmento poblacional). En efecto, se trata de un modo particular de dotar de forma a las relaciones sociales - que no son, en el fondo, sino comunicaciones - a partir de una distinción que las ordene y pretenda introducirse (negociada o conflictivamente) como legítima y vinculante para todos los afectados por ella.

El momento de lo político es el de la "demanda contestataria" (Estrada Saavedra, 20I5: 285ss.) que, al inaugurar un conflicto, cuestiona las formas de orden y, por tanto, de inclusión/exclusión del sistema. Esta demanda se basa en una interpretación alternativa (en ocasiones contrapuesta) a la manera de enmarcar y procesar temas y problemas por parte de la política. Así, tiene lugar una lucha antagónica por imponer hegemónicamente una u otra interpretación sobre el objeto de disputa. A partir de un trazo distintivo diferente - es decir, una nueva interpretación - se reordena la parcela en cuestión del mundo social con sus jerarquías, formas de acción y comunicación legítimas, pero también con sus exclusiones, categorización de grupos y personas, imágenes de sí mismo, etcétera. 
La distinción la política/lo político permite pensar, asimismo, la diferencia de los espacios y tiempos en los que los fenómenos en su interior se constituyen. Esto significa que los lugares y momentos institucionales del sistema político no agotan las formas y procesos espacio-temporales de lo político. No hay coincidencia ni sincronía, punto por punto, en las arenas de conflicto de ambos. De tal suerte, en cada uno de los lados de la distinción se generan y despliegan múltiples lógicas comunicativas - o, en términos no sistémicos, prácticas y discursos - que, empero y eventualmente, pueden traducirse entre sí, pero que, en su paso de un lado a otro, sufren transformaciones.

Al configurarse en el extrarradio de la ciudadela política, lo político alimenta, por otro lado, sus comunicaciones de los lenguajes y experiencias no tecnificados de la vida cotidiana y de las necesidades, problemas y demandas que los actores enfrentan en este ámbito de la realidad. En este sentido, lo político no representa ninguna ruptura con la vida cotidiana y el mundo de vida. De hecho, es un fenómeno más común y extendido que lo que los filósofos tienden a suponer. Efectivamente, lo político toma con frecuencia las experiencias individuales y colectivas de la vida cotidiana como el sustrato desde el cual se configura él mismo. Los actores tienen, por lo general, una idea razonablemente clara acerca de sus problemas y de lo que se requiere hacer para resolverlos. Sin embargo, las instituciones políticas no están preparadas para responder a sus necesidades por no encajar en su visión, lógica y recursos presupuestales. Justo en este desencuentro se gestan las condiciones del conflicto político.

\section{CONCLUSIÓN}

En este trabajo he afirmado que los paradigmas centrales en el estudio de los movimientos sociales resultan insuficientes para comprender su dimensión política. Por esta razón, he propuesto superar este déficit introduciendo la distinción la política/lo político. Abordar el tema de lo político tomando como punto de entrada a los movimientos sociales, tiene una doble ventaja heurística. En primer lugar, debido a que los movimientos se pueden ubicar en el interior o el exterior del sistema político, dependiendo del momento e interés de la observación, nos vemos obligados a cuestionar la identificación convencional de la política con lo estatal y a preguntarnos por su eidos. Y, en segundo término, los conflictos en los que se hallan involucrados los movimientos fungen como un dispositivo que develan la arbitrariedad de las distinciones que ordenan la vida sociopolítica y que se fundamentan en la naturalización de las relaciones de dominación. En este sentido, el empleo de la distinción la política/ lo político nos puede ayudar a des-estatizar nuestra concepción del fenómeno y a acceder a nuevos horizontes de comprensión.

El sociólogo interesado en el estudio empírico de fenómenos políticos - como los movimientos sociales, por ejemplo - hará bien en tomar conciencia 
de que la distinción la política/lo político es únicamente un instrumento heurístico, que demuestra su utilidad al permitir observar dichos fenómenos cruzando, constantemente, de un lado al otro de la distinción. No tiene por qué permanecer en el lado de la política, como prescribe el teórico sistémico, o de lo político, como quiere el filósofo. Ninguno de estos flancos ha de tomarse como más fundamental o auténtico que su contraparte.

Recibido en I9/9/20I9 | Revisado en I3/I/2020 | Aprobado en 2I/I/2020

Marco Estrada Saavedra es profesor-investigador adscrito al Centro de Estudios Sociológicos de El Colegio de México. Entre sus últimos libros se encuentran El uno y los muchos. Voluntad y soberanía en la filosofía política de Hobbes, Rousseau, Schmitt, Agamben y Arendt (2019) y Contornos de lo político. Ensayos sociológicos sobre memoria, protesta, violencia y Estado (20I9). También es autor del artículo "The Popular Assembly of the Peoples of Oaxaca (APPO)" in The Oxford Research Encyclopedia of Latin American History (2020) 


\section{NOTAS}

I Deseo agradecer a David Luján Verón, Gabriel Vommaro, Sergio Pignuoli Ocampo y a los dictaminadores anónimos de Sociologia \& Antropologia por sus comentarios, sugerencias y críticas hechas a diferentes versiones de este artículo. Me han resultado muy estimulantes.

2 Para un panorama sobre los diversos enfoques en el estudio de los movimientos sociales y la acción colectiva, véase Hellmann y Koopmans (I998), McAdam, McCarthy, y Zald (2005) y Snow, Della Porta, Klandermas y McAdam (2013).

3 Esto es especialmente cierto en la obra de Alain Touraine, Francesco Alberoni y Alberto Melucci, pero solo parcialmente correcto en la de Claus Offe o Jürgen Habermas.

4 Para un tratamiento más detallado sobre los supuestos ontológicos y epistemológicos de estos paradigmas y para una crítica de sus alcances explicativos, véase Estrada Saavedra (20I5), en especial capítulos I a 3 .

5 Debido a que este tema lo he desarrollado ampliamente en otras publicaciones (Estrada Saavedra, I995 y 20I5), aquí no abundaré más al respecto.

6 Para una exposición sinóptica de su sociología política, consúltese Hellmann (2002, 2004 y 2005). Discusiones críticas en torno a la concepción luhmanniana del sistema político, se encuentran en los tomos colectivos editados por Hellmann y Schmalz-Bruns (2002), y Hellmann, Fisher y Bluhm (2003).

7 Una introducción comparativa de la descripción de Luhmann de los sistemas funcionales de la política, el derecho, la religión, el arte, la educación, los medios de masas, entre otros, se encuentra en el tomo colectivo editado por Runkel y Burkart (2005).

8 Por ejemplo, para la persona que viola el reglamento que prohíbe fumar en locales públicos como los restaurantes.

9 Este podría ser el caso de quien, mediante la desobediencia civil, protesta contra una ley que considera injusta o de quien comete actos considerados como terroristas por la legislación penal en cuestión.

Io $\mathrm{Al}$ menos esta fue la última versión del código político ofrecida por Luhmann en su obra póstuma Die Politik der 
Gesellschaft. En este mismo libro también se mencionan el código poder superior/poder inferior y el esquema izquierda/derecha (Luhmann, 2000a: 88s, 95s y 99ss). Sobre los déficits y problemas de la concepción del código político en Luhmann, léase Hellmann (2005), en particular 29ss.

I I Esta diferenciación la trato más adelante.

I 2 Cfr. Luhmann (200ob).

I3 Para una discusión crítica de los conceptos "público", “opinión pública” y "espacio público”, revísese Görcke (2003) y Fuhse (2003).

I4 El poder judicial es, en la sociología de Luhmann, parte del sistema del derecho de la sociedad. Sin embargo, presta sus servicios al sistema político mediante acoplamientos estructurales, que se pueden ubicar, por ejemplo, en las operaciones de la suprema corte de justicia o la corte constitucional.

I5 Para la concepción de Luhmann de los "movimientos de protesta", véase Luhmann (I996), Hellmann (I996) y Estrada Saavedra (2012).

I6 Formal e institucional significan aquí, simplemente, vinculado a un cargo público, por ejemplo, funcionario o empleado público, diputado, subsecretario de gobierno, etcétera.

I7 Sólo hay que imaginar la miríada de decisiones tomadas constantemente en cualquiera de los subsistemas de la política para darse cuenta de la complejidad y variedad interna del sistema político. Tomar conciencia de esto, nos pone a salvo de la ilusión sobre la existencia de un control central y panóptico - jel soberano en la filosofía política moderna temprana!- de la política. La inexistencia de un centro supervisor y una racionalidad omnívora y omnipresente en el sistema, no significa, por supuesto, que no hay diferencias de poder y capacidad de decisión al interior de la política.

I8 Piénsese, por ejemplo, cómo el clientelismo político abreva, a la vez, de lógicas de redes informales y de las diferenciaciones sociales de la estratificación y la funcional. A manera de ejemplo, léase Luján (2017), y Luján y Pérez (20I8). Tomar conciencia de esta multiplicidad de lógicas 
coproductoras de los fenómenos políticos debería ser suficiente para evitar el prejuicio de ver en la diferenciación funcional la clave maestra de explicación de la fenomenología política en la sociedad contemporánea.

I9 Sobra decir que, en las páginas de este artículo, no me es posible tratar cada una de estas filosofías con la extensión y el detalle debidos. Sobre la discusión contemporánea en torno a lo político, consúltense Estrada Saavedra (2002), Marchart (2010), Bröckling \& Feustel (2010) y Hebekus \& Völker (20I2).

20 El artículo de Luhmann citado lleva el título "Das Ende der alteuropäischen Politik" (I988), publicado en el Tijdschrift voor de Studie van de Verlichtung en va het Vrije Denken, I6e Jaargang, 2/4, p. 249.

2I Más allá de esta polémica, para Arendt, Lefort o Castoriadis, los horrores del totalitarismo nazi y comunista fueron la razón del inicio de sus reflexiones sobre la naturaleza de la política y sus posibilidades en condiciones postotalitarias. En cambio, para pensadores más contemporáneos como Vollrath, Rancière, Badiou, Mouffe o Laclau, su interés por lo político surge de su crítica al funcionamiento y organización del orden político de las democracias realmente existentes.

22 En especial, la comunitarista, la liberal y la de impronta kantiana, es decir, las corrientes más influyentes en el discurso filosófico de los últimos 50 años.

23 Sobre el tema, véase Crouch (2015), Blündorn (2013), Michelsen y Walter (2013), y Offe (20I6).

24 Piénsese en la acción y la deliberación mediante intercambio de juicios en Arendt, o en los criterios de modalidad de la acción y de su percepción en Vollrath.

25 Por ejemplo, el espacio público y el espacio de aparición de Arendt.

26 En las oposiciones policía-política de Rancière y Estadopolítica de Badiou, por ejemplo, en la que lo político (que los franceses denominan "la política") tiene un carácter disruptivo del funcionamiento normal de las instituciones.

27 La idea arendtiana de que el fin de la política es la libertad, o también la concepción de la "regla de partición y distribución” del orden de Rancière, por ejemplo. 
$28 \mathrm{El} \mathrm{momento} \mathrm{instituyente} \mathrm{y} \mathrm{creativo} \mathrm{de} \mathrm{lo} \mathrm{social} \mathrm{en} \mathrm{Casto-}$ riadis o la idea del poder como "espacio vacío" en Lefort.

29 Por ejemplo, la relación amigo-enemigo de acuerdo con Carl Schmitt o, más contemporáneamente, los antagonismos según Laclau y Mouffe.

30 Es decir, la diferencia política/político.

3I Uno se siente tentado a reformular los valores del código, en términos más clásicos, como dominación/obediencia, acaso sólo para hacer más visible la violencia subyacente a toda relación política.

32 Topográficamente hablando, lo político se puede ubicar, a la vez, por dentro y por fuera del sistema político. Su lugar es el del umbral, en donde desestabiliza las fronteras mediante la inclusión de lo excluido.

\section{REFERENCIAS BIBLIOGRÁFICAS}

Abrams, Philip. (I988) [1977]. Notes on the difficulty of studying the State. Journal of Historical Sociology, I/I, p. 5889.

Agudo Sanchíz, Alejandro. (20I4). El Estado, disgregado y reconstituido. In: Agudo Sanchíz \& Estrada Saavedra (cords.). Formas reales de dominación del Estado. Perspectivas interdisciplinarias del poder y la política. Ciudad de México: El Colegio de México, p. 9-5I.

Agudo Sanchíz, Alejandro \& Estrada Saavedra, Marco (coords.). (20I4). Formas reales de dominación del Estado. Perspectivas interdisciplinarias del poder y la política. Ciudad de México: El Colegio de México.

Agudo Sanchíz, Alejandro \& Estrada Saavedra, Marco (eds.). (20II). (Trans)formaciones del Estado en los márgenes de América Latina. Imaginarios alternativos, aparatos inacabados y espacios transnacionales. Ciudad de México: El Colegio de México/Universidad Iberoamericana.

Agudo Sanchíz, Alejandro; Estrada Saavedra, Marco \& Braig, Mariane (coords.). (2017). Estatalidades diversas y soberanías disputadas. La reorganización contemporánea del poder y la autoridad en América Latina. Ciudad de México: El Colegio de México/Freie Universität Berlin. 
Alberoni, Francesco. (1984). Movimiento e institución. Madrid: Editorial Nacional.

Arendt, Hannah. (1994). Über die Revolution. München: Piper.

Arendt, Hannah. (I993). Was ist Politik? München: Piper.

Arendt, Hannah. (1992). Vita activa oder Vom tätigen Leben. München: Piper.

Arendt, Hannah. (1985). Das Urteilen. Texte zu Kants politischer Philosophie. München: Piper.

Badiou, Alain. (I988). L'être et l'événement. Paris: Seuil.

Badiou, Alain. (1985). Peut-on penser la politique? Paris: Seuil.

Bedorf, Thomas. (2010). Das Politische und die Politik Konturen einer Differenz. In: Bedorf, Thomas \& Röttgers, Kurt. Das Politische und die Politik. Berlin: Suhrkamp, p. 13-37. Bedorf, Thomas \& Röttgers, Kurt (2010). Vorwort. In: Bedorf, Thomas \& Röttgers, Kurt. Das Politische und die Politik. Berlin: Suhrkamp, p. 7-Io.

Blündorn, Ingolfur. (2013). Simulative Demokratie. Neue Politik nach der postdemokratischen Wende. Berlin: Suhrkamp. Bonacker, Thorsten. (2005). Die Konfliktheorie der autopoietischen Systemtheorie. In: Bonacker, Thorsten (Hrsg). Sozialwissenschatliche Konfliktheorien. Eine Einführung. Wiesbaden: VS Verlag, 3 Auflage, p. 267-292.

Bourdieu, Pierre. (I999). Meditaciones pascalianas. Barcelona: Anagrama.

Bourdieu, Pierre. (I997). Razones prácticas. Sobre la teoría de la acción. Barcelona: Anagrama.

Bröckling, Ulrich \& Feustel, Robert. (20I0). Einleitung: Das Politische denken. In: Bröckling, Ulrich \& Feustel, Robert (eds.). Das Politische denken. Zeitgenössische Positionen. Bielefeld: Transcript, p. 7-I8.

Castoriadis, Cornelius. (2008). Poder, política, autonomía. In: Un mundo fragmentado. La Plata: Terramar Ediciones, p. 87-II4.

Castoriadis, Cornelius. (200I). Imaginario e imaginación en la encrucijada. In: Figuras de lo pensable. Ciudad de México: Fondo de Cultura Económica, p. 93-II4. 
Castoriadis, Cornelius. (I995). Lo imaginario: la creación en el dominio histórico social. In Los dominios del hombre. Las encrucijadas del laberinto. Madrid: Gedisa, p. 64-80.

Castoriadis, Cornelius. (I989). La institución imaginaria de la sociedad. V.2. El imaginario social y la institución. Barcelona: Tusquets.

Clam, Jean. (2002). Was heisst, sich an Differenz statt an Identität orientieren? Zur De-ontologisierung in Philosophie und Sozialwissenschaft. Konstanz: UVK Verlagsgesellschaft.

Cohen, Jean. (I985). Strategy or identity: new theoretical paradigms and contemporary social movements. Social Research, 52/4, p. 663-716.

Cohen, Jean \& Arato, Andrew. (2000). Sociedad civil y teoría política. Ciudad de México: FCE.

Crouch, Colin. (2015) [2003]. Postdemokratie. I 2 Auflage. Frankfurt am Main: Suhrkamp.

Das, Veena \& Poole, Deborah (ed.). (2004). Anthropology in the margins of the state. Santa Fe: School of American Research Press.

Eribon, Didier. (2017). Rückkehr nach Reims. I6 Auflage. Berlin: Suhrkamp.

Estrada Saavedra, Marco. (2019). Cómo colocarle el anzuelo a la ballena. Apuntes sobre la antropología del Estado. In: Estrada Saavedra. Contornos de lo político. Ensayos sociológicos sobre memoria, protesta, violencia y Estado. Ciudad de México. El Colegio de México, p. I69-ıgo.

Estrada Saavedra, Marco. (20I6). El pueblo ensaya la revolución. La APPO y el sistema de dominación oaxaqueño. Ciudad de México: El Colegio de México.

Estrada Saavedra, Marco. (20I5). Sistemas de protestas. Esbozo de un modelo no accionalista de los movimientos sociales. Ciudad de México. El Colegio de México.

Estrada Saavedra, Marco. (2012). Riesgo, miedo y protesta: los movimientos sociales en la obra de Niklas Luhmann. In: Estrada Saavedra, Marco (coord.). Protesta social. Tres estudios sobre movimientos sociales en clave de la teoría de sistemas de Niklas Luhmann. Ciudad de México: El Colegio de México, p. 23-43.

Estrada Saavedra, Marco. (2007). La comunidad armada rebelde y el EZLN. Un estudio histórico y sociológico sobre las 
bases de apoyo zapatistas en las cañadas tojolabales de la selva lacandona (1935-2005). Ciudad de México: El Colegio de México.

Estrada Saavedra, Marco. (2002). Die deliberative Rationalität des Politischen. Eine Interpretation der Urteilslehre Hannah Arendts. Würzburg: Königshausen \& Neumann.

Estrada Saavedra, Marco. (I997). ¿Es reformable la teoría de los actores colectivos?. Revista Mexicana de Sociología, 59/3, p. 25-57.

Estrada Saavedra, Marco. (I995). Participación política y actores colectivos. Ciudad de México: Universidad Iberoamericana/Plaza y Valdés.

Fuhse, Jan. (2003). Das widerständige Publikum. Zur Relevanz von alltäglichen Kommunikationsstrukturen für die politische Meinungsbildung. In: Hellmann, Karl-Uwe; Fisher, Karsten \& Bluhm, Harald (Hrsg.). Das System der Politik. Niklas Luhmanns Politische Theorie. Wiesbaden: Westdeutscher Verlag, p. I36-I49.

Görcke, Alexander. (2003). Das System der Massmedien, öffentlche Meinung und Öffentlichkeit. In: Hellmann, Karl-Uwe; Fisher, Karsten \& Bluhm, Harald (Hrsg.). Das System der Politik. Niklas Luhmanns Politische Theorie. Wiesbaden: Westdeutscher Verlag, p. I2I-I35.

Gripp-Hagelstange, Helga. (2004). Niklas Luhmann o: ¿en qué consiste el principio teórico sustentado en la diferencia. In: Luhmann, Niklas. La política como sistema. Edición y traducción a cargo de Javier Torres Nafarrate. Ciudad de México: FCE/UIA/UNAM, p. I9-4I.

Habermas, Jürgen. (I989). Teoría de la acción comunicativa. Complementos y estudios previos. Madrid: Cátedra.

Habermas, Jürgen. (I988). Teoría de la acción comunicativa, t. I y II. Madrid: Taurus.

Hebekus, Uwe \& Völker, Jan. (2012a). Einleitung. In: Hebekus, Uwe \& Völker, Jan. Neue Philosophien des Politischen. Zur Einführung. Hamburg: Junius, p. 9-28.

Hellmann, Karl-Uwe. (2005). Spezifyk und Autonomie des politischen Systems. Analyse und Kritik der politischen Soziologie Niklas Luhmann. In: Runkel, Gunter \& Burkart, Günter (Hrsg.). Funktionssyteme der Gesellschaft. Beiträge zur Systemtheorie von Niklas Luhmann. Wiesbaden: VS Verlag 
für Sozialwissenchaften, p. I3-5I.

Hellmann, Karl-Uwe. (2004). Aristóteles y nosotros. In: Luhmann, Niklas. La política como sistema. Edición y traducción a cargo de Javier Torres Nafarrate. Ciudad de México: FCE/UIA/UNAM, p. 43-92.

Hellmann, Karl-Uwe. (2002). Einleitung. In: Hellmann, Karl-Uwe \& Schmalz-Bruns, Rainer (Hrsg.). Theorie der Politik. Niklas Luhmanns politische Soziologie. Frankfurt am Main: Suhrkamp, p. II-37.

Hellmann, Kai-Uwe. (1996). Systemtheorie und neue soziale Bewegungen. Identitätsprobleme in der Risikogesellschaft. Opladen: Westdeutscher Verlag.

Hellmann, Karl-Uwe; Fisher, Karsten \& Bluhm, Harald. (2003). Einleitung: Niklas Luhmanns politische Theorie in der politikwissenschaftlichen Diskussion. In: Hellmann, Karl-Uwe; Fisher, Karsten \& Bluhm, Harald (Hrsg.). Das System der Politik. Niklas Luhmanns Politische Theorie. Wiesbaden: Westdeutscher Verlag, p. 9-I8.

Hellmann, Kai-Uwe \& Koopmans, Rudolf (Hrsg.). (I998). Paradigmen der Bewegungsforschung. Entstehung und Etwicklung von neuen sozialen Bewegungen und Rechtextremismus. Opladen/Wiesbaden: Westdeutscher Verlag.

Hellmann, Karl-Uwe \& Schmalz-Bruns, Rainer (Hrsg.). (2002). Theorie der Politik. Niklas Luhmanns politische Soziologie. Frankfurt am Main: Suhrkamp.

Holz, Claus. (2003). Politik und Staat. Differenzierungstheoretische Probleme in Niklas Luhmanns Theorie des politischen Systems. In: Hellmann, Karl-Uwe; Fisher, Karsten \& Bluhm, Harald (Hrsg.). Das System der Politik. Niklas Luhmanns Politische Theorie. Wiesbaden: Westdeutscher Verlag, p. 34-48.

Joseph, Gilbert M. \& Nugent, Daniel (eds.). (I994). Everyday forms of state formation. Revolution and the negotiation of rule in modern Mexico. Durham/London: Duke University Press.

Krupa, Christopher \& Nugent, David (eds.). (2015). State theory and Andean politics new approaches to the study of rule. Philadelphia: University of Pennsylvania Press.

Kuhn, Thomas. (1979). La estructura de las revoluciones científicas. Ciudad de México: FCE. 
Laclau, Ernesto. (20I5). La razón populista. 3ํㅜㄹ reimpresión. Buenos Aires: FCE.

Laclau, Ernesto. (20I4). Los fundamentos retóricos de la sociedad. Buenos Aires: FCE.

Laclau, Ernesto. (2008). Debates y combates. Por un nuevo horizonte de la política. Io reimpresión. Buenos Aires: FCE. Laclau, Ernesto \& Mouffe, Chantal. (20II). Hegemonía y estrategia socialista. Hacia una radicalización de la democracia. 3o edic., Io reimpresión. Buenos Aires: FCE.

Latour, Bruno. (20I0). Eine neue Soziologie für eine neue Gesellschaft. Frankfurt am Main: Suhrkamp.

Lefort, Claude. (I999). Complications. Paris: Librairie Arthème Fayard.

Lefort, Claude. (1992). Écrire. À l'épreuve du politique. Paris: Foundation Saint-Simmon/Calmann-Levy.

Lefort, Claude. (I99I). L'invention démocratique. Les limits de la domination totalitaire. Paris: Ed. Fayard.

Lefort, Claude. (I986). Essai sur le politique. Paris: Editions du Seuil.

Lehmann, Maren. (2003). Anmerkungen zur Differenz Inklusion/Exklusion. In: Hellmann, Karl-Uwe; Fisher, Karsten \& Bluhm, Harald (Hrsg.). Das System der Politik. Niklas Luhmanns Politische Theorie. Wiesbaden: Westdeutscher Verlag, p. I63-I78.

Luhmann, Niklas. (20I2). Macht im System. Berlin: Suhrkamp.

Luhmann, Niklas. (2010). Politische Soziologie. Berlin: Suhrkamp.

Luhmann, Niklas. (2000a). Die Politik der Gesellschaft. Frankfurt am Main: Suhrkamp.

Luhmann, Niklas. (200ob). La realidad de los medios de ma sas. Barcelona: Universidad Iberoamericana/Anthropos.

Luhmann, Niklas. (2000c). Organisation und Entscheidung. Wiesbaden: Westedeutscher Verlag.

Luhmann, Niklas. (1998). Die Gesellschaft der Gesellschaft. Frankfurt am Main: Suhrkamp.

Luhmann, Niklas. (1996). Protest. Systemtheorie und soziale Bewegungen. Herausgegeben und eingeleitet von Kai-Uwe Hellmann. Frankfurt am Main: Suhrkamp. 
Luhmann, Niklas. (1987). Soziale Systeme. Grundriss einer allgemeinen Theorie. Frankfurt am Main: Suhrkamp.

Luján, David. (2017). Entre la autonomía y la dependencia. Lobby, clientelismo político e idea de estado en los contextos locales chilenos. Polis, 48, p. I33-I54.

Luján, David \& Pérez, Aníbal. (2018). Confianza, favor, lealtad. Clientelismo en dos municipalidades chilenas. Revista Sociológica, 34, p. 235-168.

McAdam, Doug; McCarthy, John D. \& Zald, Mayer N. (eds.). (2005). Comparative perspectives on social movements. Political opportunities, mobilizing structures, and cultural framings. 7th printing. New York: Cambridge University Press.

Marchart, Oliver. (2013). Das unmögliche Objekt. Eine postfundamentalistische Theorie der Gesellschaft. Berlin: Suhrkamp.

Marchart, Oliver. (2010). Die politische Differenz. Zum Denken des Politischen bei Nancy, Lefort, Badiou, Laclau und Agamben. Berlin: Suhrkamp.

McAdam, Doug; Tarrow, Sidney \& Tilly, Charles. (200I). Dynamics of contentions. New York: Cambridge University Press.

Melucci, Alberto (1996). Challenging codes. Collective action in the information age. Cambridge: Cambridge University Press.

Melucci, Alberto. (1989). Nomads of the present. Social movements and individual needs in contemporary society. London: Hutchinson radius.

Michelsen, Danny \& Walter, Franz. (2013). Unpolitische Demokratie. Zur Krise der Repräsentation. Berlin: Suhrkamp.

Migdal, Joel S. (20II). Estados débiles, Estados fuertes. Ciudad de México: FCE.

Mitchell, Timothy. (I99I). The limits of the State: beyond statist approaches and their critics. American Political Science Review, 85/I, p. 77-97.

Nassehi, Armin. (2002). Politik des Staates oder Politik der Gesellschaft? Kollektivität als Problemformel des Politischen. In: Hellmann, Karl-Uwe \& Schmalz-Bruns, Rainer (Hrsg.). Theorie der Politik. Niklas Luhmanns politische Soziologie. Frankfurt am Main: Suhrkamp, p. 38-59. 
Oberschall, Anthony. (I973). Social conflict and social movements. New Jersey: Yale University/Prentice-Hall.

Offe, Claus. (2016). Europa in der Falle. Berlin: Suhrkamp. Offe, Claus. (1988). Partidos políticos y nuevos movimientos sociales. Madrid: Editorial Sistema.

Offe, Claus. (1985). New social movements. Challenging the boundaries of institutional politics. Social Research, 52/4, p. 817-869.

Olson, Mancur, Jr. (1965). The logic of collective action, public goods and the theory of groups. Cambridge/Massachusetts: Harvard University Press.

Rancière, Jacques. (2007). El odio a la democracia. Buenos Aires/Madrid: Amorrortu.

Rancière, Jacques. (2004). Aux bords du politique. Paris: Gallimard.

Rancière, Jacques. (2002). Das Unverhnemen. Politik und Philosophie. Frankfurt am Main: Suhrkamp.

Runkel, Gunter \& Burkart, Günter. (2005). Einleitung: Luhmann und die Funktionssysteme. In: Runkel, Gunter \& Burkart, Günter (Hrsg.). Funktionssyteme der Gesellschaft. Beiträge zur Systemtheorie von Niklas Luhmann. Wiesbaden: VS Verlag für Sozialwissenchaften, p. 7-II.

Sharma, Aradhana \& Gupta, Akhil (eds.). (2006). The anthropology of the State. A reader. Oxford: Blackwell.

Snow, David et al. (2013). The Wiley-Blackwell Encyclopedia of Social and Political Movements. London: Blackwell Publishing.

Tarrow, Sidney. (1977). El poder en movimiento. Los movimientos sociales, la acción colectiva y la política. Madrid: Alianza Editorial.

Tilly, Charles. (I978). From mobilization to revolution. New York: McGraw-Hill.

Touraine, Alain. (2002). Le retour de l'acteur. Essai de sociologie. Paris: Fayard.

Touraine, Alain. (I999). Sociologie de l'action. Essai sur la société industrielle. Nouvelle édition, entièrement revue. Paris: Éditions du Seuil. 
Touraine, Alain. (I995). Producción de la sociedad. Ciudad de México: IIS/UNAM.

Touraine, Alain. (I974). Pour la sociologie. Paris: Éditions du Seuil.

Vollrath, Ernst. (2003). Was ist das Politische? Eine Theorie des Politischen und seiner Wahrnehmnung. Würzburg: Königshausen \& Neumann.

Vollrath, Ernst. (1987). Grundlegung einer philosophischen Theorie des Politischen. Würzburg: Königshausen \& Neumann.

Vollrath, Ernst. (1977). Die Rekonstruktion der politischen Urteilskraft. Stuttgart: Klett Verlag. 


\section{O CONCEITO SISTÊMICO DO POLÍTICO. \\ UM ESBOÇO}

Resumo

Com base em um diálogo entre as teorias dos movimentos sociais, a antropologia do Estado, a sociologia política de Niklas Luhmann e a filosofia política contemporânea, critica-se, neste artigo, a maneira convencional pela qual se costuma descrever as dimensões políticas dos movimentos sociais. Para apreender sua rica e complexa fenomenologia, propõe-se aqui o esboço de um conceito sistêmico do político.

THE SYSTEMIC CONCEPT OF THE POLITICAL.

\section{AN OUTLINE}

Abstract

Based on a dialogue between the theories of social movements, the anthropology of the State, the political sociology of Niklas Luhmann and contemporary political philosophy, this article criticizes the conventional way in which the political dimensions of social movements have tended to be described. As an alternative that allows us to apprehend the rich and complex phenomenology of these movements, the author outlines a systemic concept of the political.

\section{Palavras-chave}

Movimentos sociais; sistemas de protesto; antropologia do Estado teoria dos sistemas sociais; filosofia política contemporânea; conflito.

\section{Keywords}

Social moviments;

protest systems;

anthropology of the State; social systems theory; philosophy of the political; conflict. 